\title{
RESEÑA
}

\section{RECENSIÓN DE LIBRO}

Bologna, E. (2012). Estadística para psicología y educación. Córdoba: Brujas. 490 p.

\author{
Luis Fernando Ramos Vargas* \\ Universidad Privada de Tacna, Perú
}

En la actualidad, la estadística se ha convertido en uno de los pilares del método científico, las posibilidades que ofrece de aplicar modelos probabilísticos para explicar la realidad a través de la incertidumbre la ha convertido en una asignatura obligada en casi todas las universidades del mundo. Su masificación ha implicado incursionar en la mayoría de áreas del conocimiento, ubicándose como parte importante de la malla curricular en un gran número de carreras profesionales. A pesar de la importancia que acarrea la estadística para la investigación, los estudios sobre su enseñanza en niveles universitarios muestran que es una de las asignaturas que presenta mayor rechazo por parte del estudiante, siendo este fenómeno más marcado en las carreras que no están relacionadas con números, como lo son aquellas dentro de las ciencias sociales, las ciencias de la educación, ciencias de la comunicación, entre otras.

Se debe tener presente que en toda situación de aprendizaje, no solamente influyen factores intelectuales, sino que también entran al proceso factores actitudinales que afectan de forma positiva o negativa al resultado del aprendizaje. El enseñar estadística para estudiantes de carreras no orientadas a los números comprende una situación especial y a su vez un reto para el docente, ya que implica iniciar un proceso de cambio actitudinal para lograr un aprendizaje verdadero en el alumno.

El libro Estadística para psicología y educación se presenta como un texto introductorio dirigido hacia personas con ningún conocimiento de la estadística, o para aquellos con algún nivel de conocimientos que deseen reforzar. A lo largo del libro se muestra de forma pragmática las aplicaciones de los distintos conceptos en situaciones reales. El libro procura centrarse en la comprensión del razonamiento que sostiene a cada procedimiento y le quita protagonismo a los cálculos matemáticos, que posiblemente sea aquello que crea mayor aversión en los estudiantes de estas carreras en el aprendizaje de la estadística. El autor, Eduardo Bologna, adjunta el software estadístico InfoStat, que es un paquete de análisis de datos desarrollado por la Universidad de Córdoba, en Argentina (para mayor información el lector puede remitirse a la página web del programa). 
Desde la presentación del libro, el autor procura responder la pregunta, quizás más consultada en los salones de clases, ¿Estadística en psicología y educación? En estas primeras páginas se expone cómo es posible que la estadística pueda aplicarse en estas áreas del conocimiento, la explicación es desarrollada con gran maestría y precisión, se busca que el lector comprenda cómo el estudio desde lo colectivo puede explicar el comportamiento individual; el autor refiere:

“Conocer las regularidades grupales no implica dejar de lado al individuo, por el contrario, implica situarlo en relación a un grupo y conocer factores de orden general que pueden estar afectándolo individualmente" (Bologna, 2012, p. 13).

Si bien el autor explica la importancia de la aplicación de la estadística, también procura que el lector la perciba con una mirada crítica y busque la rigurosidad propia del método científico, para elaborar y leer la estadística.

En general, el libro sigue una estructura basada en las tres partes que reflejan las áreas de estudio más importantes en la estadística: la estadística descriptiva, el estudio de la probabilidad $\mathrm{y}$, por último y quizás la más importante, la estadística inferencial. La distribución por capítulos, según los apartados, está propuesta de la siguiente manera: la estadística descriptiva es abordada desde el primer hasta el quinto capítulo, el estudio de la probabilidad es desarrollado a partir del sexto hasta el octavo y la estadística inferencial se expone desde el noveno hasta el final del libro. Es importante resaltar las actividades prácticas de repaso que se incluyen al final de cada capítulo, además de las explicaciones procedimentales propuestas a través del paquete estadístico InfoStat.
La primera parte se encuentra dividida, a su vez, en dos secciones, en la primera se revisa a través de un enfoque univariado, explicando temas como la naturaleza de las variables, sus niveles de medición, las matrices de datos, la utilización de gráficos y las medidas de posición, que incluye las medidas de tendencia central y las medidas de dispersión. En el cuarto y quinto capítulo se aborda la estadística descriptiva empleando un enfoque bivariado, se revisan conceptos como la direccionalidad, la intensidad y la forma de relación entre variables. Las tablas de resúmenes de los conceptos proporcionadas por el autor, facilitan al lector su comprensión puntual de los temas desarrollados.

La segunda parte comprende el estudio de la probabilidad, el contenido de estos capítulos presenta mayor densidad en comparación con los capítulos anteriores. El autor procura utilizar solo la información necesaria para la comprensión práctica de la probabilidad, cabe resaltar la separación que realiza entre la asignación a priori y la asignación a posteriori, que sirve de gran ayuda para su entendimiento. Como texto introductorio, le brinda al lector las nociones más importantes respecto a la probabilidad, muestreo y una introducción concisa sobre la relación existente entre la población y la muestra, también el autor motiva al lector para que busque, por iniciativa propia, mayor información, para lo cual proporciona referencias bibliográficas que ayuden a profundizar esta temática.

En la tercera y última parte del libro, aborda la estadística inferencial desde una perspectiva pragmática más que teórica, lo cual es apropiado para el públicolectoral cualsedirige el texto. El autor desarrolla aquellas pruebas estadísticas con mayor uso en la investigación psicológica y educativa, principalmente se centra en la estadística inferencial bivariada, 
aunque también presenta menciones de técnicas multivariadas. Es útil el repaso de lo leído para verificar aquellos supuestos necesarios para el correcto funcionamiento de los procedimientos utilizados. El texto también comprende un espacio para introducir las pruebas no paramétricas y, al igual que en el capítulo anterior, la redacción concisa facilita la comprensión de lo expuesto. Al final del libro, el autor presenta el trabajo de un colaborador para instruir al lector en el uso del InfoStat.

El autor, Eduardo Bologna, al ser psicólogo y docente universitario comprende muy bien las actitudes desfavorables que presenta un gran número de estudiantes universitarios de psicología y educación en el aprendizaje de la estadística, por lo que a lo largo del texto se puede apreciar la concisión, la claridad y el pragmatismo que impregna el autor al momento de explicarla. Considerando que, muchas veces, el primer vistazo que el estudiante percibe de la aplicación del método científico se da en un curso introductorio de estadística, el docente debe ser un facilitador para su acercamiento, y textos como el reseñado, estoy seguro que mejora la experiencia tanto del estudiante como del propio docente.

(c) Los autores. Este artículo es publicado por la Revista Digital de Investigación en Docencia Universitaria del Área de Investigación de la Dirección de Calidad Educativa, Universidad Peruana de Ciencias Aplicadas. Este es un artículo de acceso abierto, distribuido bajo los términos dela Licencia Creative Commons Atribución-CompartirIgual4.0 Internacional.(http://creativecommons.org/licenses/by-sa/4.0/), que permite el uso no comercial, distribución y reproducción en cualquier medio, siempre que la obra original sea debidamente citada. 